Wien klin Mag 2020 • 23:131-139

https://doi.org/10.1007/s00740-020-00339-z

Online publiziert: 22. April 2020

(c) Springer-Verlag GmbH Austria, ein Teil von Springer Nature 2020

Jedes Jahr erkranken in Deutschland mehr als 450.000 Menschen neu an Krebs [1]. In den letzten 20 Jahren hat sich die Überlebensrate von Krebspatienten in Deutschland durch die verbesserten Therapiemodalitäten deutlich erhöht. Infektionen stellen bei onkologischen Patienten weiterhin eine relevante Ursache für Mortalität und auch Morbidität dar, die mit längeren Krankenhausaufenthalten und steigenden Kosten einhergehen $[2,6]$.

\section{Hämatologische und onkologische Besonderheiten}

Im Vergleich zu anderen Patientengruppen haben Patienten mit hämatologischen und onkologischen Erkrankungen ein erhöhtes Risiko für infektiöse Komplikationen.

》) Der häufigste Notfall in dieser Patientengruppe ist die febrile Neutropenie

Der häufigste Notfall in dieser Patientengruppe ist die febrile Neutropenie mit einem relevanten Sepsis- und Mortalitätsrisiko. Im vorliegenden Artikel wird auch auf weitere Infektionen eingegangen, die sich ebenfalls zum Notfall entwickeln können. Neben der chemotherapieinduzierten Neutropenie sind verschiedene Risikofaktoren für die Entwicklung von Infektionen zu nennen:

Dieser Beitrag wurde in der Zeitschrift Der Onkologe 2/2020 26:129-138 https://doi.org/ 10.1007/s00761-019-00691-8 erstpubliziert. Zweitpublikation mit freundlicher Genehmigung der Autoren.

Andreas Edwin Juarso - Malte Kiehl · Markus Buchholz · Florian Weißinger

Klinik für Innere Medizin, Hämatologie, Onkologie, Stammzelltransplantation und Palliativmedizin, Evangelisches Klinikum Bethel, Bielefeld, Deutschland

\title{
Infektionen als Notfall der Onkologie
}

\section{Übersicht über die aktuellen Leitlinien}

- tumorbedingte Obstruktion des respiratorischen, gastrointestinalen oder urogenitalen Trakts [3],

- Störung der Schleimhautbarriere,

- Veränderungen der mikrobiologischen Flora,

- Defekte in der humoralen und zellulären Immunität,

- Störungen der Phagozytose,

- Splenektomie als Teil der Therapie, tumorassoziierte Immunsuppression,

- Steroidtherapie,

- Einsatz von Venenverweilkathetern.

Die Dosisintensität der Chemotherapie korreliert mit der Dauer und dem Nadir der Neutropenie, welche wiederum mit der Inzidenz von Fieber und Infektionen eng verbunden ist [4].

Aufgrund der häufig dosisintensiveren Therapieprotokolle und der meist erkrankungs- oder therapiebedingten Immunsuppression besteht bei hämatologischen Patienten in der Regel eine höhere Wahrscheinlichkeit, eine Neutropenie zu entwickeln, als bei Patienten mit soliden Tumoren. Daher sind infektiöse Komplikationen in dieser Patientengruppe häufiger und für mehr als die Hälfte der Todesfälle verantwortlich $[3,5]$.

\section{Beispiele infektiöser Komplikationen}

\section{Fieber in Neutropenie}

Fieber in Neutropenie stellt einen Notfall bei onkologischen Patienten dar. Fieber kann das einzige Symptom einer Infektion bei diesen Patienten sein. Die Beurteilung des Schweregrads der Infektion wird bei noch klinisch unauffällig erscheinen- den Patienten häufig unterschätzt. Grund dafür ist die defekte Wirtsverteidigungslage, sodass typische Entzündungsreaktionen fehlen können. Eine Therapieverzögerung ist allerdings bei bestehenden Komorbiditäten mit einer Mortalität von mehr als 50\% verbunden [6], daher ist die unverzügliche Erkennung und $\mathrm{Be}$ handlung des Fiebers in Neutropenie sehr wichtig.

Neutropenie wird von den meisten Leitlinien als eine absolute neutrophile Zahl (stab- und segmentkernige Granulozyten) von $<500 / \mu$ l oder $<1000 / \mu l \mathrm{mit}$ zu erwartender Abnahme bis $<500 / \mu l$ in den folgenden 2 Tagen definiert. Als Fieber gilt entweder eine einmalige oral gemessene Körpertemperatur von $\geq 38,3^{\circ} \mathrm{C}$ oder eine Körpertemperatur von mindestens $38,0^{\circ} \mathrm{C}$, die mindestens $1 \mathrm{~h}$ dauert oder innerhalb von $12 \mathrm{~h} 2$-malig nachgewiesen wird $[4,7]$.

\section{》) Die klinische Beurteilung durch das behandelnde Ärzteteam spielt eine wichtige Rolle}

Im Allgemeinen werden febrile Neutropenien, die mehr als 8 Tage dauern, als High-Risk-Erkrankung eingestuft. Neben der Schwere der Neutropenie tragen mehrere Faktoren zu der Morbidität und Mortalität der hämatoonkologischen Patienten bei. So werden die Kriterien gemäß der Multinational Association of Supportive Care in Cancer (MASCC) häufig angewendet, um eine Stratifikation der Patientenrisiken zu ermöglichen und um $\mathrm{zu}$ entscheiden, ob eine am- 


\begin{tabular}{|c|c|}
\hline Kriterien & Punkte \\
\hline Schweregrad der febrilen Neutropenie ohne oder mit leichten Symptomen & 5 \\
\hline Keine Hypotonie (systolischer Blutdruck >90 mm Hg) & 5 \\
\hline Keine chronische obstruktive Lungenerkrankung & 4 \\
\hline $\begin{array}{l}\text { Solide Tumoren oder hämatologische Malignität ohne stattgehabte Fungalinfek- } \\
\text { tionen }\end{array}$ & 4 \\
\hline Keine Dehydratation mit Notwendigkeit parenteraler Flüssigkeit & 3 \\
\hline Schweregrad der febrilen Neutropenie mit mäßig ausgeprägten Symptomen & 3 \\
\hline Ambulante Behandlung & 3 \\
\hline Alter $<60$ Jahre & 2 \\
\hline
\end{tabular}

Tab. 2 Kriterien gemäß Clinical Index of Stable Febrile Neutropenia (CISNE)

\begin{tabular}{l|l}
\hline Kriterien & Punkte \\
\hline Performance-Status gemäß Eastern Cooperative Oncology Group: $\geq 2$ & 2 \\
\hline Chronische obstruktive Lungenerkrankung & 1 \\
\hline $\begin{array}{l}\text { Chronische kardiovaskuläre Erkrankungen } \\
\text { Mukositis des Grades } \geq 2 \text { nach National Cancer Institute Common Toxicity Criteria }\end{array}$ & 1 \\
\hline $\begin{array}{l}\text { Monozytenzahl } \leq 200 / \mu l \\
\text { Stressinduzierte Hyperglykämie }\end{array}$ & 1 \\
\hline $\begin{array}{l}\text { Gesamtpunktezahl von } 0 \text { bis } 8 \\
\text { 0 Punkte - Niedrigrisikopatient } \\
1-2 \text { Punkte - Mittelrisikopatient } \\
\geq 3 \text { Punkte - Hochrisikopatient }\end{array}$ & 2 \\
\hline
\end{tabular}

bulante oder stationäre Behandlung indiziert ist (• Tab. 1).

Die aktuelle Datenlage weist daraufhin, dass der MASCC-Score allein nicht ausreichend ist, um alle Patienten mit Komplikationen $\mathrm{zu}$ erkennen. In einer gepoolten Analyse entwickelten sich bei $\geq 11 \%$ der Patienten, die mit dem MASCC-Score als Niedrigrisikopatienten klassifiziert wurden, ernsthafte Komplikationen. Andere Faktoren wie fortgeschrittenes Stadium, febrile Neutropenie in der Vorgeschichte, fehlende antibiotische Prophylaxe oder Gabe von "granulocyte colony stimulating factor" (G-CSF), „poor performance status“ und kardiovaskuläre Begleiterkrankungen sind daher auch zu berücksichtigen. Ein weiterer Score, der Clinical Index of Stable Febrile Neutropenia (CISNE) wird von der ASCO (American Society of Clinical Oncology) empfohlen, um eine bessere Triage der Patienten zu ermöglichen. Letztendlich spielt die klinische Beurteilung durch das behandelnde Ärzteteam eine wichtige Rolle, um das Risiko der Situation einschätzen zu können und über die stationäre nen die Symptome sehr subtil sein. Auf veränderte Vigilanz, Unwohlsein der Patienten, Hypotonie, und subfebrile oder afebrile Temperaturen ist $\mathrm{zu}$ achten, da diese auf eine gramnegative Sepsis hindeuten könnten und eine sofortige Therapie benötigen.

Neben den in der klinischen Routine üblichen Laboruntersuchungen, wie Blutbild, Gerinnungswerte, Leber- und Nierenfunktion, C-reaktives Protein, Laktatdehydrogenase (LDH) und Urinstatus, sind mindestens 2 Blutkulturen, z. B. aus beiden Armen, unerlässlich. Wurde bei dem Patienten ein zentraler Venenkatheter (ZVK) gelegt, sollen Blutkulturen, am besten aus jedem Katheterlumen, abgenommen werden. Eine Differenz von $\pm 2 \mathrm{~h}$ in Umschlagzeiten zwischen den zentralen und peripheren Blutkulturen könnte mit hoher Wahrscheinlichkeit auf einen katheterbedingten Infekt hinweisen. Während einer prolongierten Neutropeniephase ist die regelmäßige Durchführung der genannten routinemäßigen Blutuntersuchungen, z. B. 2-mal in der Woche, $\mathrm{zu}$ empfehlen. Die Bestimmung von Laktat, Blutgasanalyse und Gerinnung kann zur Erkennung einer Sepsis dienen. Daten für die Bestimmung weiterer Entzündungsmarker wie Procalcitonin und Interleukin-6 zur Verlaufskontrolle sind jedoch umstritten $[4,8]$.

\section{》) Hochrisikopatienten sollen stationär aufgenommen werden und eine i.v.-Antibiose erhalten}

In der Gruppe der Niedrigrisikopatienten könnte eine orale antibiotische Therapie möglich sein, vorausgesetzt, dass der Patient sich im hämodynamisch stabilen Zustand befindet, keine akute Leukämie oder ein Endorganversagen hat, und dass Infektionen wie Pneumonie, Verweilkatheter- und/oder schwerer Weichteilinfekt ausgeschlossen sind [7]. Hochrisikopatienten sollen dagegen stationär aufgenommen werden und eine i.v.-Antibiose erhalten. Die frühzeitige empirische Gabe einer Antibiotikatherapie, unmittelbar nach der Abnahme von Blut- und Urinkulturen, wird in den meisten Leitlinien hervorgehoben. Dabei soll die Therapie 
Hier steht eine Anzeige.

\section{曾 Springer}


unverzüglich, innerhalb von $1 \mathrm{~h}$ nach der Erstvorstellung und spätestens $2 \mathrm{~h}$ nach Beginn des Fiebers, eingesetzt werden, ohne Abwarten des mikrobiologischen Befundes [4, 7, 8]. Die erhaltene prophylaktische Therapie mit Chinolonen soll dann abgesetzt werden. In • Tab. 3 wird die empfohlene antibiotische Therapie zusammengefasst.

Die kombinierte antibiotische Therapie ist bei der aktuellen Datenlage der antibiotischen Monotherapie nicht überlegen. Auch zeigte die kombinierte antibiotische Therapie bezüglich der Resistenzentwicklung keinen Vorteil [9]. Diese könnte jedoch bei erhöhter Prävalenz multiresistenter Keime in Betracht gezogen werden. Dabei bestünde die Kombination aus $\beta$-Laktam-Antibiotika mit Antipseudomonasaktivität und z. B. mit Aminoglykosiden. In Fällen von schwerer Mukositis, Haut- oder Weichteilinfektionen, Fremdkörperinfektionen oder nachgewiesenem MRSA (methicillinresistentem Staphylococcus aureus) könnte eine Kombination mit Vancomycin, Teicoplanin oder Linezolid verwendet werden [4]. Das therapeutische Drugmonitoring mittels Medikamentenspiegel soll bei dem Einsatz von Vancomycin aufgrund des erhöhten Risikos einer $\mathrm{Ne}$ phrotoxizität durchgeführt werden.

Bei Patienten mit nachgewiesener Besiedlung durch vancomycinresistente Enterokokken kann derzeit die Hinzunahme von Linezolid aufgrund des fehlenden Vorteils und des Thrombopenierisikos nicht empfohlen werden [4].

\section{Febrile Neutropenie mit Lungeninfiltraten}

Lungeninfiltrate können bei bis zu 25\% der Patienten mit schwerer und prolongierter Neutropenie nachgewiesen werden [10]. $\mathrm{Zu}$ den möglichen kausalen pathologischen Keimen gehören Aspergillus spp., Pneumocystis jirovecii, Mycoplasma pneumoniae, Chlamydia pneumoniae, Legionellen, Nocardia asteroides, multiresistente gramnegative Bakterien, Mykobakterien, respiratorische Viren sowie Fadenpilze.

Bei Patienten mit febriler Neutropenie und respiratorischen Symptomen ist die Mehrschicht- oder hochauflösende

Wien klin Mag 2020 - 23:131-139 https://doi.org/10.1007/s00740-020-00339-z

(c) Springer-Verlag GmbH Austria, ein Teil von Springer Nature 2020

\section{A. E. Juarso · M. Kiehl · M. Buchholz · F. Weißinger}

\section{Infektionen als Notfall der Onkologie. Übersicht über die aktuellen Leitlinien}

\section{Zusammenfassung}

Hintergrund. Patienten mit onkologischen Erkrankungen, insbesondere mit hämatologischen Neoplasien, haben ein erhöhtes Risiko für allgemeine Infektionen und spezielle therapieassoziierte Komplikationen mit hoher Morbidität und Mortalität. Die jährliche Inzidenz und Prävalenz für Krebserkrankungen steigt in Deutschland. Moderne Therapieverfahren haben zu einem verbesserten Überleben geführt, jedoch erfordern steigendes Alter, Komorbiditäten und Gebrechlichkeit der Patienten multidisziplinäre Strategien zur Durchführung komplexer Therapiekonzepte und zur Behandlung von deren Komplikationen. Methode. Eine selektive Literaturrecherche sowie die Leitlinien der European Society for Medical Oncology (ESMO), der Deutschen Gesellschaft für Hämatologie und Medizinische Onkologie (DGHO), der Arbeitsgemeinschaft Infektionen in der Hämatologie und Onkologie der DGHO
(AGIHO) und der American Society of Clinical Oncology (ASCO) sind die Grundlage für den vorliegenden Artikel.

Schlussfolgerungen. Das Erkennen schwerer Infektionen bei Krebspatienten und deren Unterscheidung von therapieassoziierten Komplikationen stellt eine Herausforderung für den behandelnden Arzt dar. Neutropenisches Fieber ist der häufigste infektiologische Notfall in der Onkologie. Frühzeitige empirische Therapie mit Breitspektrumantibiotika und eskalierende diagnostische Maßnahmen sind notwendig für die erfolgreiche Therapie dieser Risikogruppe. In diesem Beitrag wird eine Reihe von lebensbedrohlichen Infektionen bei immunkompromittierten Patienten diskutiert.

Schlüsselwörter

Hämatologische Neoplasien · Sepsis · ZNSInfektionen - Immunsuppression - Mukositis . Fieber in Neutropenie

\section{Infections as oncologic emergencies. Overview of current guidelines}

\section{Abstract}

Background. Patients with oncologic diseases, particularly those with hematologic malignancies, are at an increased risk of common infections and unique treatmentrelated complications with high mortality and morbidity. The annual incidence and prevalence of cancer in Germany is rising. Although modern treatments have generally led to improved survival, increasing age, comorbidities, and frailty of the patients require multidisciplinary strategies for handling complex therapeutic concepts and treatment of the associated complications. Methods. A selective literature search and guidelines from the European Society for Medical Oncology (ESMO), the German Society of Hematology and Medical Oncology (Deutsche Gesellschaft für Hämatologie und Medizinische Onkologie, DGHO), the Infectious Diseases Working Group of the DGHO (Arbeitsgemeinschaft Infektionen in der
Hämatologie und Onkologie, AGIHO), and the American Society of Clinical Oncology (ASCO) formed the basis of this study. Conclusion. Recognition of severe infections in cancer patients and their discrimination from treatment-associated complications is a challenge. Neutropenic fever is the most frequent infectious emergency in oncology. Early empiric treatment with broadspectrum antibiotics and escalated diagnostic strategies are needed to successfully treat this vulnerable patient group. In this article, a range of potentially life-threatening infections in immunocompromised patients are discussed.

\section{Keywords}

Hematologic neoplasms · Sepsis · CNS infections - Immunosuppression · Mucositis . Neutropenic fever 
Tab. 3 Antibiotische Therapie für den Niedrigrisiko- und Hochrisikopatienten mit febriler Neutropenie

\begin{tabular}{|c|c|c|}
\hline & Niedrigrisikopatient & Hochrisikopatient \\
\hline $\begin{array}{l}\text { Erste } \\
\text { Linie }\end{array}$ & $\begin{array}{l}\text { Ambulante Behandlung: } \\
\text { Amoxicillin/Clavulansäure } \\
\text { Bei Penicillinallergie: } \\
\text { Clindamycin + Ciprofloxacin } \\
\text { Cefuroxim + Ciprofloxacin } \\
\text { Moxifloxacin } \\
\text { Stationäre Behandlung: } \\
\text { Ceftazidim } \\
\text { Cefepim } \\
\text { Piperacillin/Tazobactam } \\
\text { Beim Nachweis multiresistenter gramne- } \\
\text { gativer Bakterien: } \\
\text { 3./4. Generation der Cephalospori- } \\
\text { ne + Aminoglykoside }\end{array}$ & $\begin{array}{l}\text { Piperacillin/Tazobactam } \\
\text { Cefepim, Ceftazidim } \\
\text { Bei Penicilinallergie: } \\
\text { Imipenem, Meropenem } \\
\text { Cephalosporine mit Antipseudomonas- } \\
\text { aktivität }\end{array}$ \\
\hline $\begin{array}{l}\text { Zweite } \\
\text { Linie }\end{array}$ & $\begin{array}{l}\text { Bei Versagen der ambulanten und oralen } \\
\text { antibiotischen Therapie: } \\
\text { Piperacillin/Tazobactam } \\
\text { Imipenem, Meropenem }\end{array}$ & $\begin{array}{l}\text { Nach Piperacillin/Tazobactam oder Cefe- } \\
\text { pim oder Ceftazidim: } \\
\text { Imipenem, Meropenem } \\
\text { Nach Imipenem oder Meropenem: } \\
\text { Hinzunahme von Vancomycin oder Tei- } \\
\text { coplanin oder Aminoglykosiden plus } \\
\text { gegen Schimmelpilz wirksames Antimy- } \\
\text { kotikum }\end{array}$ \\
\hline
\end{tabular}

Computertomographie der Lunge das diagnostische Mittel der Wahl. Bei den meisten Patienten ist die Anwendung von Kontrastmittel nicht erforderlich. Alternativ kann die Durchführung einer Magnetresonanztomographie vorgenommen werden. Die Diagnostik soll innerhalb von $24 \mathrm{~h}$ nach Indikationsstellung stattfinden ([7, 10], • Abb. 1).

\section{》) Die Diagnostik soll innerhalb von $24 \mathrm{~h}$ nach Indikationsstellung stattfinden}

Beim positiven Nachweis von Infiltraten soll eine bronchoalveoläre Lavage, aus dem das auffällige Areal versorgenden Segmentbronchus, erfolgen. Auch diese soll innerhalb von $24 \mathrm{~h}$ nach Indikationsstellung durchgeführt werden, sofern keine kritische Hypoxämie vorliegt. Die Aufarbeitung der bronchoalveolären Lavage umfasst die Polymerasekettenreaktion (PCR) für Mycobacterium tuberculosis, PCR und direkte Immunfluoreszenz für Pneumocystis jirovecii, Aspergillusantigen (Galactomannan-ELISA, „enzyme-linked immunosorbent assay") sowie Bakterienkulturen inklusive Legionella spp. und Mykobakterien. Außerdem werden auch PCR für Zytomegalievirus
(CMV), Respiratory-Syncytial-Virus, Influenza A/B, Parainfluenza 1-3, Metapneumovirus, Adenovirus sowie Varizella-

Eine antimykotische Therapie soll beim Nachweis von Infiltraten begonnen werden, die nicht typisch für eine Pneumocystispneumonie oder eine bakterielle Lobärpneumonie sind. Therapie der Wahl ist Voriconazol oder liposomales Amphotericin B [11]. Beim bildgebenden Verdacht auf Pneumocystispneumonie und einer neu aufgetretenen LDH-Erhöhung soll eine Therapie mit hochdosiertem Trimethoprim-Sulfamethoxazol auch vor der Durchführung einer bronchoalveolären Lavage erfolgen. Nach einer erfolgreichen Therapie sollen die Patienten eine orale Sekundärprophylaxe erhalten. Bei Patienten mit Nachweis von Pseudomonas aeruginosa sollte eine Therapie mit pseudomonaswirksamen $\beta$-Laktamen wie Piperacillin/Tazobactam, Ceftazidim, Imipenem/ Cilastatin, Meropenem oder Cefepim in Kombination mit einem Aminoglykosid verwendet werden. Bei Kontraindikationen gegen Aminoglykoside könnte stattdessen Ciprofloxacin zum Einsatz kommen [7, 10].

In Fälle der auftretenden respiratorischen Insuffizienz soll eine intensivmeZoster-Virus empfohlen. dizinische Behandlung unter einem multidisziplinären Expertenteam zur Verfügung stehen.

\section{Invasive Pilzinfektionen}

Persistierendes Fieber $\geq 96$ h oder Rezidiv des Fiebers bei neutropenischen Patienten unter antibiotischer Therapie könnte ein Hinweis auf Pilzinfektion darstellen. Die Möglichkeit einer invasiven Pilzinfektion erfordert hohe Aufmerksamkeit, da diese mit signifikanter Morbidität und Mortalität einhergeht [2]. Zu Beginn steht eine empirische antimykotische Therapie zur Verfügung. Bei Patienten, die schon prophylaktisch Voriconazol oder Posaconazol erhielten und keine klinischen Hinweise auf invasive Pilzinfektionen zeigen, ist eine Umstellung der antimykotischen Therapie nicht empfohlen. Bei Fieberpersistenz, klinischer Verschlechterung oder Instabilität ist eine Umstellung aufCaspofungin oder liposomales Amphotericin B indiziert [4, 12].

Eine Wiederholung der CT-Untersuchung mit Durchführung einer Aspergillus-Galaktomannan-PCR ist bei persistierendem Fieber gerechtfertigt, da bei einem positiven Befund eine spezifische antimykotische Therapie (in Einzelfällen auch inklusive chirurgischer Resektion) notwendig sein können [13].

\section{Sepsis bei neutropenischen Patienten}

Die Mortalität der neutropenischen $\mathrm{Pa}$ tienten, die sich im septischen Schock befinden, ist mit einem Wert von bis zu $50 \%$ sehr hoch [6]. Die tägliche Untersuchung von Patienten mit Neutropenie und Infektzeichen spielt daher eine sehr wichtige Rolle für die frühzeitige Erkennung von Zeichen einer Infektionsquelle und möglichen Sepsisursache. Die Diagnose von Sepsis und septischem Schock unterscheidet nicht zwischen den neutropenischen und nichtneutropenischen Patienten. Die Definition der Sepsis umfasst den Score „quick Sepsis-related Organ Failure Assessment“ (qSOFA) und den Nachweis von Organdysfunktionen. Bei Notwendigkeit der Gabe von Vasopressoren, um einen mittleren arteriellen 


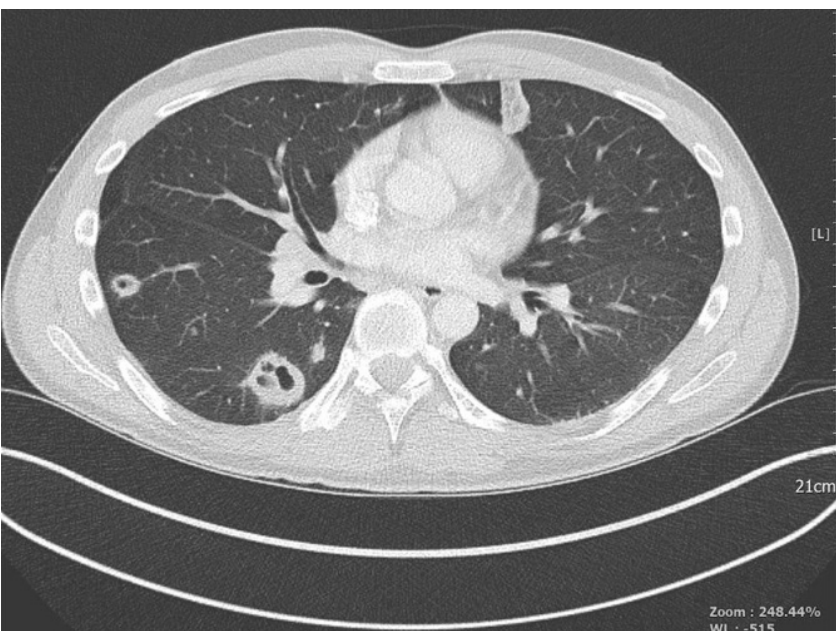

Abb. $1 \Delta$ Bild einer Aspergillusinfektion der Lunge bei einem Patienten mit akuter Leukämie

Druck von $\geq 65 \mathrm{~mm} \mathrm{Hg}$ zu erreichen, trotz adäquater Flüssigkeitsgabe, und Serumlaktat von $\geq 2 \mathrm{mmol} / \mathrm{l}$ ist die Diagnose septischer Schock gesichert [14].

Im Allgemeinen sollen beim Nachweis von Sepsis/septischem Schock die Behandlungsziele, Prognose und der Patientenwillen geklärt werden. Neutropenische Patienten mit Indikation zur intensivmedizinischen Behandlung sollen akut - und nicht verzögert - auf die Intensivstation aufgenommen werden. Dies gilt auch für unklare klinische Situationen, deren weitere Einschätzung erst im Verlauf der intensivmedizinischen Therapie geklärt werden kann. Multidisziplinäre Konsultationen zwischen den behandelnden Onkologen und Intensivmedizinern sollen täglich erfolgen, um die Therapieplanung festzulegen und ggf. die Umsetzung des Behandlungskonzepts zu prüfen.

\section{》) Eine antibiotische Therapie mit pseudomonaswirksamen Antibiotika ist bei Sepsis sofort einzuleiten}

Eine empirische antibiotische Therapie mit pseudomonaswirksamen Antibiotika ist bei Sepsis sofort einzuleiten. Mittel der Wahl sind Piperacillin/Tazobactam, Meropenem oder Imipenem/Cilastatin [15]. Bei septischem Schock kann eine Kombination mit einem Aminoglykosid er-

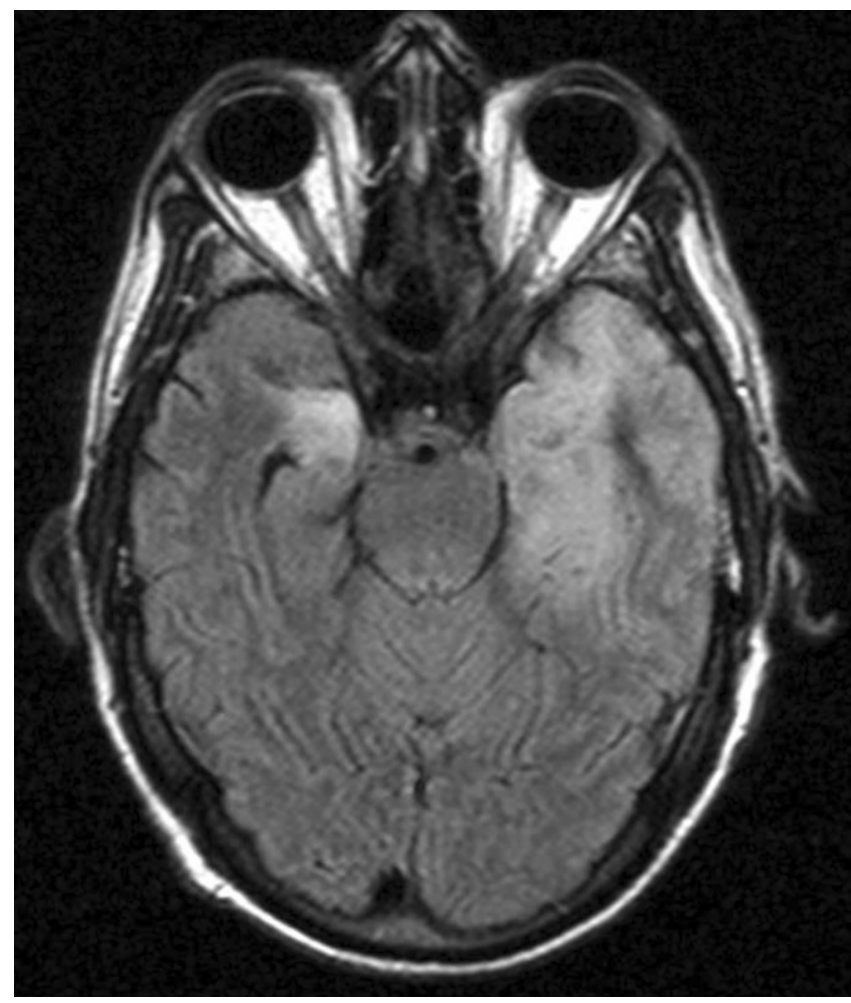

Abb. 2 \ Bild einer Herpesenzephalitis links temporomesial bei einem immunsupprimierten Patienten

wogen werden. Die Therapie mit Aminoglykosid kann im Verlauf bei Stabilisierung des Patienten oder Nachweis von $\beta$-Laktam-sensitiven Keimen abgesetzt werden. Bei persistierender kardiorespiratorischer Instabilität ist die Erweiterung mit einer antimykotischen Therapie zu erwägen [16]. Die Therapie mit Kortikosteroiden ist von unklarer Bedeutung und ist individuell je nach Schwere der Sepsis sowie der hämatoonkologischen Grunderkrankung zu entscheiden.

Die Suche nach einer möglicher Infektquelle soll nicht verzögert werden, auch wenn dafür ein chirurgischer Eingriff oder eine CT-gesteuerte Punktion benötigt wird. Bezüglich der Flüssigkeitssubstitution, Verwendung von Katecholaminen, Transfusion von Blutprodukten, Reanimationsmaßnahmen, Sedierung und Analgesie, mechanischer Beatmung, Physiotherapie, Blutzuckerkontrolle, Nierenersatztherapie, Bikarbonatgabe und Stressulkusprophylaxe gibt es keinen Unterschied zu den nichtneutropenischen Patienten [16]. Zur Thromboseprophylaxe wird derzeit die Anwendung von unfraktioniertem oder niedermolekularem Heparin empfohlen, solange keine Kontraindikation vorliegt. Weder Granulozytensubstitution noch G-CSF als ergänzende Therapie bei Sepsis und septischem Schock werden empfohlen, zumal das Risiko einer respiratorischen Verschlechterung mit Entwicklung eines ARDS (,adult respiratory distress syndrome") bei G-CSFinduzierter Erholung der Granulozyten nach einer Neutropeniephase besteht.

\section{ZNS-Infektionen}

Infekte des zentralen Nervensystems (ZNS) stellen bei allen Patienten einen Notfall da. Für Patienten mit hämatologischen und onkologischen Erkrankungen, v. a. Patienten mit Zustand nach allogener Transplantation, besteht mit bis $\mathrm{zu} 15 \%$ eine signifikante Inzidenz von ZNS-Infektionen [17]. Auslösende Erreger sind in $\bullet$ Abb. 2 dargestellt.

Die Diagnosestellung einer ZNSInfektion kann eine große Herausforderung darstellen, da klinische und neurologische Symptome unspezifisch sind oder durch Nebenwirkungen von 
Chemotherapie oder Immunsuppressiva hervorgerufen werden können. Ferner ist die Differenzierung zwischen ZNSInfektion, Grunderkrankung (z. B. ZNSMetastasen) und Begleiterkrankungen (wie progressive multifokale Leukoenzephalopathie bei Patienten mit HIV, Humanimmundefizienzvirus) nicht einfach. Zur Diagnosestellung werden mindestens eine neuroradiologische Bildgebung (bevorzugt MRT), eine Liquordiagnostik und eine eventuelle Biopsie verdächtiger Herde benötigt. Zu berücksichtigen sind typische Infektionen bei spezifischen Patientengruppen (z.B. Kryptokokkenmeningitis oder zerebrale Toxoplasmose bei vorhandenem Defekt der T-Zell-Immunität oder verminderter Makrophagenfunktion) sowie lokale endemische pathologische Erreger, wie Histoplasma capsulatum, Mycobacterium tuberculosis und Toxoplasma spp. (• Tab.4).

\section{Gastrointestinale Komplikationen}

Die Inzidenz gastrointestinaler Komplikation ist bei onkologischen Patienten deutlich erhöht und involviert je nach Therapie unterschiedlich stark verschiedene Bereiche des Gastrointestinaltrakts (von Mundhöhle bis Analregion). Im oberen Gastrointestinaltrakt treten insbesondere nach kombinierter Radiochemotherapie oder modernen zielgerichteten Therapien wie z. B. Tyrosinkinaseinhibitoren, mTOR-Inhibitoren („mammalian target of rapamycin“) oder Antikörper Fälle von Mukositis, Candida- und/oder Herpesinfektionen auf $[7,18]$. Bei schwerer Mukositis ist häufig eine parenterale Ernährung erforderlich, die jedoch das Risiko sekundärer Katheterinfektionen birgt $[18,19]$.

\section{》) Bei schwerer Mukositis ist häufig eine parenterale Ernährung erforderlich}

Bei vesikulären labialen oder enoralen Läsionen sollte vor Beginn der empirischen Therapie mit Aciclovir eine Probe entnommen werden. Die Therapie mit Ganciclovir (oder Foscarnet) ist nur bei hochgradigem Verdacht auf eine Infektion mit CMV indiziert [7].
Im unteren Gastrointestinaltrakt stellen bei Symptomen wie Diarrhö und Kolitis - neben der Therapie derselben - v. a. die Diagnostik und deren Abgrenzung zu Nebenwirkungen antineoplastischer Therapien und moderner zielgerichteter Therapien (z.B. Tyrosinkinaseinhibitoren und Immuncheckpointinhibitoren) die führenden Probleme dar [20-22]. Bei Diarrhöen ( $\geq 3$ weiche Stühle/24h) sollte initial die Stuhlbeurteilung hinsichtlich Farbe, Konsistenz und Beimengungen von Blut oder putriden Materials erfolgen. Anamnestisch gilt der vorherigen Einnahme von Antibiotika und weiterer Medikationen (Protonenpumpeninhibitoren) besonderes Augenmerk [21, 22].

Als supportive Maßnahme muss eine ausreichende Zufuhr von Flüssigkeit und Elektrolyten erfolgen. Die Entscheidung zur parenteralen Ernährung erfolgt abhängig von weiteren Risikofaktoren (Komorbiditäten, Mangelernährungsrisiko) $[21,22]$.

Die initiale diagnostische Aufarbeitung sollte infektiöse von nichtinfektösen Ursachen bei Durchfällen trennen. Bei neutropener Enterokolitis kann jedoch empirisch frühzeitig eine Therapie mit einem Breitspektrumantibiotikum eingeleitet werden (Piperacillin/Tazobactam, Imipenem/Cilastin oder Meropenem) [22]. Bei Clostridium-difficile-assoziierter Diarrhö steht zur Behandlung Vancomycin und Fidaxomicin an erster Stelle. Der Toxin B bindende Antikörper Bezlotoxuzumab kann in Einzelfällen zur Sekundärprophylaxe genutzt werden. Metronidazol ist in der Behandlung nur von geringem Stellenwert und sollte nicht mehr routinemäßig verabreicht werden [22].

Die Therapie der Diarrhöen durch andere bakterielle Ursachen (nichttyphöse Salmonella spp., Shigella spp., Campylobacter spp., Yersinia spp.) umfassen Ciprofloxacin und Ceftriaxon. Carbapenem oder Azithromycin kann bei Diarrhöen durch Shigatoxin produzierende E. coli erwogen werden. Virale Ursachen einer Gastroenteritis werden primär supportiv behandelt, jedoch kann bei schwerer Adenovirusenteritis Cidofovir erwogen werden. Nach Nachweis einer CMVEnteritis sollte die Behandlung mit Ganciclovir eingeleitet werden $[21,22]$.
Hepatitis (fulminante Verlaufsform)

Eine Hepatitis-B-Infektion verläuft meist schwerer als eine Hepatitis-C-Infektion [26]. Ein Risiko zur Reaktivierung einer Hepatitis B besteht prinzipiell bei jeder Art von Immunsuppression. Ein besonders Risiko konnte für verschiedene $\mathrm{Zy}$ tostatika nachgewiesen werden: 5-Fluorouracil (5-FU), Cisplatin, Anthrazykline, Vincaalkaloide, Methotrexat, Cyclophosphamid, Etoposid und Everolimus nachgewiesen werden [24]. Grundsätzlich wird die Reaktivierungsrate bei soliden Erkrankungen unterschätzt. Klinisch kann sich das Bild eines fulminanten Leberversagens mit Ikterus, Koagulopathie, Fieber und Enzephalopathie entwickeln.

Im Vergleich wurde in Studien gezeigt, dass eine prophylaktische einer therapeutischen antiviralen Behandlung überlegen ist, um Leberversagen zu verhindern. Mit einer prophylaktischen antiviralen Strategie kann auch die Intensität der antitumoralen Therapie ohne relevante Unterbrechung verhindert werden [23, 25, 26]. Für hämatologische Patienten unter R-CHOP-Therapie (Rituximab, Cyclophosphamid, Hydroxydaunorubicin, Vincristin, z. B. Oncovin ${ }^{\circledR}$, Prednison) mit positivem Nachweis des HBs-Antigens wurde die Überlegenheit einer Prophylaxe mit Entecavir im Vergleich zu Lamivudin nachgewiesen [27]. Es wurden mit Entecavir weniger HBV-Infektions-Reaktivierungen beobachtet. Das gesamte Therapiespektrum reicht von der beschriebenen konservativ-medikamentösen Behandlung bis zur Option der Lebertransplantation.

\section{Myokarditis}

Die Myokarditis ist eine heterogene Krankheitsentität mit variablem Krankheitsbild von asymptomatisch bis fulminant. Bei onkologischen Patienten ist neben einer infektiösen Genese auch eine therapieassoziierte Genese durch 5-FU, Rituximab und auch Immuntherapeutika möglich [28]. Für Immuntherapeutika wird sie als seltene Nebenwirkung bei Ipilimumab, Nivolumab, Pembrolizumab, Atezolizumab, Avelumab und Durvalumab beschrieben [29]. Als infektiöse 
Tab. 4 Auslösende Erregern der ZNS-Infektionen bei hämatoonkologischen Patienten

\begin{tabular}{|c|c|}
\hline Prädisponierende Faktoren & Pathologische Keime \\
\hline $\begin{array}{l}\text { Splenektomie } \\
\text { Antikörpermangel (multiples } \\
\text { Myelom, CLL) }\end{array}$ & $\begin{array}{l}\text { Streptococcus pneumoniae, Haemophilus influenzae, Neisseria } \\
\text { meningitides }\end{array}$ \\
\hline $\begin{array}{l}\text { Chemotherapie, Stammzell- } \\
\text { transplantation, Immunsup- } \\
\text { pression }\end{array}$ & Cryptococcus neoformans, Listeria monocytogenes \\
\hline $\begin{array}{l}\text { Defekt der T-Zell-Immunität, } \\
\text { T-Zell-Abnahme }\end{array}$ & $\begin{array}{l}\text { Histoplasma capsulatum, Mycobacterium tuberculosis, Toxoplas- } \\
\text { ma spp., Varicella-Zoster-Virus, Herpes-simplex-Virus, Zytome- } \\
\text { galovirus, JC-Virus }\end{array}$ \\
\hline
\end{tabular}

Ursache sind v. a. Viren der Gruppe Enteroviren, Adenoviren, Influenzaviren, humanes Herpesvirus 6 (HHV-6), Epstein-Barr-Virus, CMV, Hepatitis-CVirus und Parvovirus B 19 in Betracht zu ziehen. Aber auch Bakterien, Protozoen und Pilze können Auslöser sein. Zur Diagnosestellung sind Echokardiographie, Kardio-MRT und serologische Untersuchung hilfreich. Für die Sicherung der Diagnose wird eine endomyokardiale Biopsie empfohlen [30]. Die Therapie variiert je nach Pathogen und Symptomen.

\section{Katheterinfektionen}

In vielen Situationen ist bei onkologischen Patienten die Anlage eines zentralvenösen Zugangs (nichtgetunnelt/ getunnelt, PICC/,peripherally inserted central venous catheter", Port) indiziert oder hilfreich [19, 31].

Um die Diagnose Katheterinfektion festzustellen, müssen folgende Kriterien erfüllt sein: positiver Blutkulturbefund aus dem ZVK mit einer Umschlagszeit $\geq 2 \mathrm{~h}$ vor Umschlagszeit des peripheren Kulturbefundes, DTTP („differential time to positivity“), oder eine 3-fache Menge an Kolonien in ZVK-Kulturen [32].

\section{》) Bei klinischer Stabilität besteht die Möglichkeit, den Katheter zu erhalten}

Die Entfernung des Katheters sollte zwingend bei klinischer Instabilität oder einem Nachweis von Staphylococcus aureus oder Candida spp. erfolgen [19, 32].
Bei klinischer Stabilität besteht die Möglichkeit, den Katheter zu erhalten. Hierfür wird via „antibiotic lock“ (ALT) der Katheter entsprechend dem Kulturbefund mit einem Antibiotikum geblockt. Bei grampositiven Befunden kommt häufig Vancomycin, bei gramnegativen Gentamycin und bei Pilzen Amphotericin B zum Einsatz [19, 32].

Die initiale systemische antibiotische Therapie mit Vancomycin oder Teicoplanin verbessert das Outcome nicht und sollte nach kulturellem Befund resistenzgerecht eingesetzt werden [32].

\section{Fazit für die Praxis}

- Die Erkennung eines Infekts bei onkologischen Patienten und die Abgrenzung von anderen therapieassoziierten Nebenwirkungen ist eine Herausforderung.

- Die frühzeitige Einleitung der Therapie mit einem empirischen Breitbandantibiotikum ist entscheidend für das Überleben der Patienten, die sich in der Notfallsituation der febrilen Neutropenie befinden.

- Persistierendes Fieber erfordert eine Reevaluation der Ursache und Behandlung.

- Sepsis bei Neutropenie weist eine hohe Mortalität auf.

- Das diagnostische und therapeutische Vorgehen, inklusive intensivmedizinischer Maßnahmen, sind deshalb weitgehend identisch wie bei nichtneutropenischen Patienten.

- Frühzeitige bildgebende Untersuchungen sind beim Verdacht auf eine Infektion wichtig, um die weitere Abklärung und Therapie einzuleiten.

\section{Korrespondenzadresse}

Univ.-Prof. Dr. Florian Weißinger

Klinik für Innere Medizin, Hämatologie,

Onkologie, Stammzelltransplantation und

Palliativmedizin, Evangelisches Klinikum Bethel

Schildescher Str. 99, 33611 Bielefeld,

Deutschland

florian.weissinger@evkb.de

Danksagung. Wir danken dem Institut für diagnostische und interventionelle Radiologie (Prof. Dr. Günther Wittenberg) und dem Institut für diagnostische und interventionelle Neuroradiologie (Prof. Dr. Randolf Klingebiel) für die Überlassung der CT- bzw. MRT-Bilder.

\section{Einhaltung ethischer Richtlinien}

Interessenkonflikt. A.E. Juarso, M. Kiehl, M. Buchholz und F. Weißinger geben an, dass kein Interessenkonflikt besteht.

Für diesen Beitrag wurden von den Autoren keine Studien an Menschen oder Tieren durchgeführt. Für die aufgeführten Studien gelten die jeweils dort angegebenen ethischen Richtlinien.

\section{Literatur}

1. Robert Koch-Institut und die Gesellschaft der epidemiologischen Krebsregister in Deutschland e.V. (Hrsg) (2013) Krebs in Deutschland für 2013/2014. 11. Ausgabe Robert Koch-Institut (Hrsg.) und die Gesellschaft derepidemiologischen Krebsregister in Deutschland, Berlin. https://doi. org/10.17886/rkipubl-2017-007. ISBN 978-389606-288-8

2. Gary LH, Poniewiersky MS, Crawford JDDC, Culakova E (2015) Cost of hospitalization in patients with cancer and febrile neutropenia and impact of comorbid conditions. Blood 126:2089

3. Cantwell L, Perkins J (2018) Infectious disease emergencies in oncology patients. Emerg Med Clin North Am. https://doi.org/10.1016/j.emc.2018.06. 009

4. Heinz WJ, Buchheidt D, Christopeit M, von Lilienfeld-Toal M, Cornely OA, Einsele $H$, Karthaus $M$, Link H, Mahlberg R, Neumann S, Ostermann H, Penack O, Ruhnke M, Sandherr M, Schiel X, Vehreschild JJ, Weissinger F, Maschmeyer G (2017) Diagnosis and empiricial treatment of fever of unknown origin (FUO) in adult neutropenic patients: guidelines of the Infectious Disease Working Party (AGIHO) of the German Society of Hematology and Medical Oncology (DGHO). Ann Hematol 96:1775-1792. https://doi.org/10.1007/s00277017-3098-3

5. Pagano L, Caira $M$, Candoni A, Offidani $M$, Fianchi L, Martino B, Pastore D, Picardi M, Bonini A, Chierichini A, Fanci R, Caramatti C, Invernizzi R, Mattei D, Mitra ME, Melillo L, Aversa F, Van Lint MT, Falcucci P, Valentini CG, Girmenia C, Nosari A (2006) The epidemiology of fungal infections in patients with hematologic malignancies: the SEIFEM-2004 Study. Haematologica 91:1068-1075

6. Kuderer NM, Dale DC, Crawford J, Cosler LE, Lyman GH (2006) Mortality, morbidity, and cost 
associated with febrile neutropenia in adult cancer patients. Cancer 106(10):2258-2266. https://doi. org/10.1002/cncr.21847

7. Klastersky J, de Naurois J, Rolston K, Rapoport B, Maschmeyer G, Aapro M, Herrstedt J, ESMO Guidelines Committee (2016) Management of febrile neutropaenia: ESMO Clinical Practice Guidelines. Ann Oncol 27(Suppl 5):v118-v111

8. Taplitz RA, Kennedy EB, Bow EJ, Crews J, Gleason C, Hawley DK, Langston AA, Nastoupil LJ, Rajotte M, Rolston K, Strasfeld L, Flowers CR (2018) Outpatient management of fever and neutropenia in adults treated for malignancy: American society of clinical oncology and infectious diseases society of America clinical practice guideline update. J Clin Oncol. https://doi.org/10.1200/JCO.2017.77.6211

9. Tamma PD, Cosgrove SE, Maragakis LL (2012) Combination therapy for treatment of infections with gram-negative bacteria. Clin Microbiol Rev 25(3):450-470. https://doi.org/10.1128/CMR. 05041-11

10. Maschmeyer G, Fernandez JC, Buchheidt D, Hamprecht A, Heußel CP, Kahl C et al (2014) Febrile Neutropenie mit Lungeninfiltraten nach intensiver Chemotherapie. https://www.onkopedia.com/ de/onkopedia/guidelines/fieber-unbekanntergenese-fuo-bei-neutropenischen-patienten/ @@guideline/html/index.html

11. Patterson TF, Boucher HW, Herbrecht R, Denning DW, Lortholary $\mathrm{O}$, Ribaud $\mathrm{P}$, Rubin $\mathrm{RH}$, Wingard JR, DePauw B, Schlamm HT, Troke $P$, Bennett JE, for the European Organization for Research and Treatment of Cancer (EORTC) Invasive Fungal Infections Group (IFIG) and the Pfizer Global Aspergillus Study Group (2005) Strategy of following voriconazole versus amphotericin B therapy with other licensed antifungal therapy for primary treament of invasive aspergillosis: impact of other therapies on outcome. Clin Infect Dis 41(10):1448-1452 (Nov)

12. Walsh TJ, Teppler H, Donowitz GR, Maertens JA, Baden LR, Dmoszynska A, Cornely OA, Bourque MR, Lupinacci RJ, Sable CA, dePauw BE (2004) Caspofungin versus liposomal amphotericin $B$ for empirical antifungal therapy in patients with persistent fever and neutropenia. $\mathrm{N}$ Engl J Med 351:1391-1402. https://doi.org/10.1056/ NEJMoa040446

13. Mousset S, Buchheidt D, Heinz W, Ruhnke M, Cornely OA, Egerer G et al (2014) Invasive Pilzinfektionen-Therapie. https://www.onkopedia.com/de/ onkopedia/guidelines/invasive-pilzinfektionentherapie/@@uideline/html/index.html

14. Singer M, Deutschman CS, Seymour CW, ShankarHari M, Annane D, Bauer M, Bellomo R, Bernard GR, Chiche J-D, Coopersmith CM, Hotchkiss RS, Levy MM, Marshall JC, Martin GS, Opal SM, Rubenfeld GD, van der Poll T, Jean-Louis $\mathrm{V}$, Angus DC (2016) The third international consensus definitions for sepsis and septic schock (sepsis-3). J Am Med Assoc 315(8):801-810. https://doi.org/ 10.1001/jama.2016.0287

15. Penack O, Becker C, Buchheidt D, Christopeit M, Kiehl M, von-Lilienfeld-Toal M, Hentrich M, Reinwald M, Salwender H, SchalkE, Schmidt-Hieber M, Weber T, Ostermann H (2014) Management of sepsis in neutropenic patients : 2014 updated guidelines from the infectious diseases working party of the german society of hematology and medical oncology (AGIHO). Ann Hematol 93(7):1083-1095

16. Kochanek $M$, Schalk $E$, von Bergwelt-Baildon $M$, Beutel K, Buchheidt $D$, Hentrich $M$ et al (2019) Sepsis bei neutropenischen Patienten. https://www.onkopedia.com/de/onkopedia/ guidelines/sepsis-bei-neutropenischenpatienten/@@guideline/html/index.html

17. Schmidt-Hieber M, Silling G, Schalk E, Heinz W, Panse J, Penack $O$ et al (2016) ZNS-Infektionen bei hämatologischen und onkologischen Erkrankungen, einschl. allogener Stammzelltransplantation. https://www. onkopedia.com/de/onkopedia/guidelines/ zns-infektionen-bei-haematologischen-undonkologischen-erkrankungen-einschl-allogenerstammzelltransplantation/@@guideline/html/ index.html

18. Peterson DE, Boers-Doets $C B$, Bensadoun $R J$, Herrstedt J, on behalf of the ESMO Guidelines Committee (2015) Management of oral and gastrointestinal mucosal injury: ESMO clinical practice guidelines for diagnosis, treatment and follow-up. Ann Oncol 26(Suppl 5):v151-v139. https://doi.org/10.1093/annonc/mdv202

19. Sousa B, Furlanetto J, Hutka M, Gouveia $P$, Wuerstlein R, Mariz JM, Pinto D, Cardoso F, on behalf of the ESMO Guidelines Committee (2015) Central venous access in oncology: ESMO clinical practice guidelines. Ann Oncol 26(Suppl 5):v168-v152. https://doi.org/10.1093/annonc/ mdv296

20. Haanen JBAG, Carbonnel F, Robert C, Kerr KM Peters S, Larkin J, Jordan K (2017) Management of toxicities from immunotherapy: ESMO clinical practice guidelines for diagnosis, treatment and follow-up. Ann Oncol 28(Suppl 4):i142-i119. https://doi.org/10.1093/annonc/mdx225

21. Bossi P, Antonuzzo A, Cherny NI, Rosengarten 0 , Pernot S, Trippa F, Schuler U, Snegovoy A, Jordan $\mathrm{K}$, Ripamonti $\mathrm{Cl}$, on behalf of the ESMO Guidelines Committee (2018) Diarrhoe in adult cancer patients: ESMO clinical practice guidelines. Ann Oncol 29(Suppl 4):126-iv. https://doi.org/10. 1093/annonc/mdy145

22. Schmidt-Hieber $M$, Bierwirth J, Buchheidt $D$, Cornely OA, Hentrich M, Maschmeyer G etal (2018) Gastrointestinale Komplikationen (Schwerpunkt: Diarrhoe und Colitis) beiPatienten mithämatologischen und onkologischen Erkrankungen. https:// www.onkopedia.com/de/onkopedia/guidelines/ gastrointestinale-komplikationen-schwerpunktdiarrhoe-und-colitis-bei-patienten-mithaematologischen-und-onkologischenerkrankungen/@@guideline/html/index.html

23. Sandherr $M$, Einsele $H$, Hebart $H$, Kahl C, Kern W, Kiehl M, Massenkeil G, Penack O, Schiel X et al (2006) Antiviral prophylaxis in patients with haematological malignancies and solid tumours: Guidelines of the Infectious Diseases Working Party (AGIHO) of the German Society for Hematologyand Oncology (DGHO). Ann Oncol 17(7):1051-1059. https://doi.org/10.1093/annonc/mdj132

24. Voican CS, Mir O, Loulergue $P$, Dhooge $M$, Brezault C, Dréanic J, Chaussade $S$, Pol S, Coriat $R$ (2016) Hepatitis B virus reactivation in patients with solid tumors receiving systemic anticancer treatment. Ann Oncol 27:2172-2184. https://doi. org/10.1093/annonc/mdw414

25. Cainelli F, Longhi MS, Concia E, Vento S (2001) Failure of lamivudine therapy for chemotherapy-induced reactivation of hepatitis B. Am J Gastroenterol 96(5):1651-1652

26. Lau GK, Yiu HH, Fong DY, Cheng HC, Au WY, Lai LS, Cheung M, Zhang HY, Lie A, Ngan R, Liang R (2003) Early is superior to deferred preemptive lamivudine therapy for hepatitis B patients undergoing chemotherapy. Gastroenterology 125(6):1742-1749
27. Huang $\mathrm{H}$, Li X, Zhu J, Ye S, Zhang $\mathrm{H}$, Wang W, Wu X, Peng J, Xu B, Lin Y, Cao Y, Li H, Lin S, Liu Q, Lin T (2014) Entecavir vs lamivudine for prevention of hepatitis $B$ virus reactivation among patients with untreated diffuse large B-cell lymphoma receiving $\mathrm{R}-\mathrm{CHOP}$ chemotherapy: a randomized clinical trial. J Am Med Assoc 312(23):2521-2530. https://doi. org/10.1001/jama.2014.15704

28. Keramida K, Parissis JT, Chioncel O, Farmakis D (2019) Cardiogenic shock in cancer. Heart Fail Rev. https://doi.org/10.1007/s10741-019-09819-9

29. Michel L, Rassaf T, Totzeck M (2019) Cardiotoxicity from immune checkpoint inhibitors. Int J Cardiol 25:10042. https://doi.org/10.1016/j.ijcha.2019. 100420

30. Caforio AL, Pankuweit S, Arbustini E, Basso C, Gimeno-Blanes J, Felix SB, Fu M, Heliö T, Heymans S, Jahns R, Klingel K, Linhart A, Maisch B, Mckenna W, Mogensen J, Pinto YM, Ristic A, Schultheiss HP, Seggewiss $H$, Tavazzi L, Thiene G, Yilmaz A, Charron P, Elliott PM, European Society of Cardiology Working Group on Myocardial and Pericardial Diseases (2013) Current state of knowledge on aetiology, diagnosis, management, and therapy of myocarditis: a position statement of the European Society of Cardiology Working Group on Myocardial and Pericardial Diseases. Eur Heart J 34(33):2636-2648. https://doi.org/10. 1093/eurheartj/eht210

31. Gallieni M, Pittiruti M, Biffi R (2008) Vascular access in oncology patients. CA Cancer J Clin 58(6):323-346. https://doi.org/10.3322/CA.2008. 0015

32. Hentrich $M$, Schalk $E$, Schmidt-Hieber $M$, Chaberny I, Mousset S, Buchheidt D et al (2015) ZVK Infektionen. Leitlinie der Deutschen Gesellschaft für Hämatologie und medizinische Onkologie (DGHO). https://www.onkopedia. com/de/onkopedia/guidelines/zvk-infektionen/ @@guideline/html/index.html 